\title{
A remark on trigonometric sums
}

\author{
by
}

Huixue LaO (Jinan)

1. Introduction and main results. Let $\mathcal{P}$ be the set of all primes, and $\mathcal{P}_{2}$ the set of integers of the form $p_{1} p_{2}$ where $p_{1}, p_{2} \in \mathcal{P}, p_{1} \neq p_{2}$. We shall write $e(\alpha)$ instead of $e^{2 \pi i \alpha}$. Let $\pi(x)$ be the number of primes up to $x$. In [3] Kátai considered the following trigonometric sums:

$$
S\left(x, \alpha \mid X_{p}\right)=\sum_{\substack{p_{1} p_{2}<x \\ p_{1}<p_{2}}} X_{p_{1}} X_{p_{2}} e\left(\alpha p_{1} p_{2}\right),
$$

where $X_{p}$ are complex numbers satisfying $\left|X_{p}\right| \leq 1$ and $p_{1}, p_{2}$ run over the prime numbers. Let

$$
\pi_{2}(x)=\sum_{\substack{p_{1} p_{2}<x \\ p_{1}<p_{2}}} 1 .
$$

Kátai showed that

$$
\max _{\substack{\left|X_{p}\right| \leq 1 \\ p \in \mathcal{P}}} \frac{S\left(x, \alpha \mid X_{p}\right)}{\pi_{2}(x)} \rightarrow 0 \quad(x \rightarrow \infty)
$$

provided that $\alpha$ is an irrational number satisfying the following condition:

Condition $\delta$. There exists $x_{0}>0$ such that for all $x \geq x_{0}$ there exists a rational number $a / q$ with $(a, q)=1$ satisfying $x^{2 / 3+\delta}<q<x^{1-\delta}$ and $|\alpha-a / q| \leq 1 / q^{2}$. Here $\delta$ is an arbitrary small positive number.

The aim of this note is to give a stronger version of Kátai's result. To this end, we recall the definition of the irrationality measure for a real number $\alpha$.

Definition 1.1. Let $\alpha$ be a real number, and let $R(\alpha)$ be the set of positive real numbers $\mu$ for which

$$
0<|\alpha-p / q|<1 / q^{\mu}
$$

2000 Mathematics Subject Classification: 11J82, 11L20.

Key words and phrases: prime numbers, irrationality measure, trigonometric sums.

This work is supported by the National Natural Science Foundation of China (Grant Nos. 10701048 and 11571107). 
has (at most) finitely many solutions $p / q$ for $q$ and $p$ integers. Then the irrationality measure of $\alpha$ is defined as

$$
\mu(\alpha)=\inf _{\mu \in R(\alpha)} \mu .
$$

If the set $R(\alpha)$ is empty, then we set $\mu(\alpha)=\infty$.

By Dirichlet's well-known rational approximation lemma, for every irrational number $\alpha$, we have $\mu(\alpha) \geq 2$. For every irrational algebraic number $\alpha$, Roth [4] proved in 1955 that $\mu(\alpha)=2$. And it is well-known that for almost all real numbers the irrationality measure is 2 .

In this note we shall prove the following theorem.

Theorem 1.1. For any irrational number $\alpha$ with $\mu(\alpha)<\infty$, we have

$$
\max _{\substack{\left|X_{p}\right| \leq 1 \\ p \in \mathcal{P}}} \frac{S\left(x, \alpha \mid X_{p}\right)}{\pi_{2}(x)} \rightarrow 0 \quad(x \rightarrow \infty) .
$$

2. Preliminaries. We need the following two lemmas.

Lemma 2.1. Let $\Lambda(n)$ be the von Mangoldt function

$$
\Lambda(n)= \begin{cases}\log p & \text { if } n=p^{\alpha}, \\ 0 & \text { otherwise. }\end{cases}
$$

If

$$
|\alpha-a / q|<1 / q^{2}, \quad(a, q)=1,
$$

then

$$
S(\alpha)=\sum_{n \leq x} \Lambda(n) e(\alpha n) \ll\left(x q^{-1 / 2}+x^{4 / 5}+x^{1 / 2} q^{1 / 2}\right)(\log x)^{4} .
$$

Proof. See Davenport [1, Chapter 25].

Lemma 2.2. Let $\alpha$ be any irrational number with $\mu(\alpha)<\infty$. Fix $\eta \in R(\alpha)$ and $0<\varepsilon<1 / 12(\eta-1)$. Then for sufficiently large $x$ we have

$$
\max _{1 \leq H \leq x^{2 \varepsilon}}\left|\sum_{p \leq x} e(H \alpha p)\right| \ll x^{1-4 \varepsilon} .
$$

Proof. First we show that for any $1 \leq H \leq x^{2 \varepsilon}$ there exist integers $a$ and $q$ such that

$$
\left|H \alpha-\frac{a}{q}\right|<\frac{1}{q x^{1-9 \varepsilon}} \quad \text { with }(a, q)=1, x^{9 \varepsilon} \leq q \leq x^{1-9 \varepsilon} .
$$

Any irrational $H \alpha$ has just one infinite simple continued fraction. Let $a / q$ and $a^{\prime} / q^{\prime}$ be the two consecutive convergents to that continued fraction such that

$$
q \leq x^{1-9 \varepsilon}<q^{\prime} .
$$


Recall the well-known property of continued fractions:

$$
\left|H \alpha-\frac{a}{q}\right|<\frac{1}{q q^{\prime}} \quad \text { with }(a, q)=1 .
$$

If $1 \leq q \leq x^{9 \varepsilon}$, then $q H \leq x^{11 \varepsilon} \leq\left(x^{1-9 \varepsilon}\right)^{12 \varepsilon}$ for sufficiently large $x$. Thus by $(2.2)$ and (2.3),

$$
\|q \alpha H\| \leq\left(q^{\prime}\right)^{-1}<\left(x^{1-9 \varepsilon}\right)^{-1} \leq(q H)^{-1 / 12 \varepsilon},
$$

where $\|\cdot\|$ denotes the distance to the nearest integer. But for $\eta \in R(\alpha)$, the inequality

$$
\|q H \alpha\|<(q H)^{1-\eta}
$$

has (at most) finitely many integer solutions $q H$. When $x$ is sufficiently large, this contradicts the choice of $\eta$ and $\varepsilon$. Thus we have $x^{9 \varepsilon} \leq q \leq x^{1-9 \varepsilon}$.

On noting (2.1), by Lemma 2.1 we have

$$
\max _{1 \leq H \leq x^{2 \varepsilon}}\left|\sum_{p \leq x} e(H \alpha p)\right| \ll\left\{x\left(x^{9 \varepsilon}\right)^{-1 / 2}+x^{4 / 5}+x^{1 / 2}\left(x^{1-9 \varepsilon}\right)^{1 / 2}\right\}(\log x)^{3} \ll x^{1-4 \varepsilon} .
$$

3. Proof of Theorem 1.1. Following the arguments of Kátai, to prove Theorem 1.1 it suffices to show that

$$
S_{1}(x, \alpha):=\sum_{\substack{p_{1} p_{2}<x \\ p_{1}<Y, p_{2}>\sqrt{x}}} X_{p_{1}} X_{p_{2}} e\left(p_{1} p_{2} \alpha\right)=o\left(\pi_{2}(x)\right),
$$

where $Y=e^{(\log x)^{1-\delta_{x}}}, \delta_{x}$ is a function of $x$ for which $\delta_{x} \rightarrow 0$, and

$$
\pi_{2}(x)=\sum_{\substack{p_{1} p_{2}<x \\ p_{1}<p_{2}}} 1 \sim \frac{x}{\log x} \log \log x .
$$

We have

$$
S_{1}(x, \alpha)=\sum_{p_{2}} X_{p_{2}} \sum_{p_{1} \leq \min \left(x / p_{2}, Y\right)} X_{p_{1}} e\left(p_{1} p_{2} \alpha\right) .
$$

Then by the Cauchy-Schwarz inequality,

$$
\left|S_{1}(x, \alpha)\right|^{2} \leq \sum_{p_{2}}\left|X_{p_{2}}\right|^{2} \sum_{p_{2}}\left|\sum_{p_{1} \leq \min \left(x / p_{2}, Y\right)} X_{p_{1}} e\left(p_{1} p_{2} \alpha\right)\right|^{2} \leq \pi(x) \sum_{1},
$$

where

$$
\sum_{1}=\sum_{\substack{p_{1}, p_{1}^{\prime} \in \mathcal{P} \\ p_{1}, p_{1}^{\prime} \leq Y}} X_{p_{1}} \bar{X}_{p_{1}^{\prime}} \sum_{\substack{x \leq p_{2} \leq x / \max \left(p_{1}, p_{1}^{\prime}\right) \\ \sqrt{2}}} e\left(\left(p_{1}-p_{1}^{\prime}\right) p_{2} \alpha\right) .
$$

From (3.3), we have

$$
\sum_{1} \leq \sum_{2}+\sum_{3}
$$


where

$$
\sum_{2}=\sum_{p_{1}=p_{1}^{\prime} \leq Y} \sum_{\sqrt{x} \leq p_{2} \leq x / \max \left(p_{1}, p_{1}^{\prime}\right)} 1 \ll \frac{x}{\log x} \sum_{p \leq Y} \frac{1}{p} \ll \frac{x}{\log x} \log \log x,
$$

and

$$
\sum_{3}=2 \sum_{p_{1}^{\prime}<p_{1} \leq Y}\left|\sum_{\sqrt{x} \leq p_{2} \leq x / \max \left(p_{1}, p_{1}^{\prime}\right)} e\left(\left(p_{1}-p_{1}^{\prime}\right) p_{2} \alpha\right)\right| .
$$

For the inner sum in (3.6), by Lemma 2.2 we have

$$
\sum_{\sqrt{x} \leq p_{2} \leq x / \max \left(p_{1}, p_{1}^{\prime}\right)} e\left(\left(p_{1}-p_{1}^{\prime}\right) p_{2} \alpha\right) \ll x^{1-4 \varepsilon}
$$

for every $p_{1} \neq p_{1}^{\prime}$ and $p_{1}, p_{1}^{\prime}<Y$. Thus

$$
\sum_{3} \ll Y^{2} x^{1-4 \varepsilon}=o\left(\pi_{2}(x)\right) .
$$

From (3.2), (3.5) and (3.8), we complete the proof of Theorem 1.1.

4. Another similar result. Recently Indlekofer and Kátai [2] proved another result about trigonometric sums

$$
S\left(x \mid \alpha ; Y_{m}, X_{p}\right)=\sum_{m_{j} p \leq x} Y_{m_{j}} X_{p} e\left(\alpha m_{j} p\right),
$$

where the $m_{j}$ are integers depending on $x$ with $m_{1}<\cdots<m_{t} \leq x^{\delta_{x}}, \delta_{x} \rightarrow 0$ as $x \rightarrow \infty, p$ runs over the primes $p \geq \sqrt{x}$, and $\left|Y_{m_{j}}\right| \leq 1,\left|X_{p}\right| \leq 1$.

Assume further that as $x \rightarrow \infty$,

$$
\sum_{j=1}^{t} \frac{1}{m_{j}} \rightarrow \infty
$$

Then they proved that provided that the irrational number $\alpha$ satisfies the Condition $\delta$ (see Section 1), we have

$$
\max _{Y_{m}, X_{p}}\left|S\left(x \mid \alpha ; Y_{m}, X_{p}\right)\right|=o_{x}(1) \sum_{j=1}^{t} \pi\left(x / m_{j}\right) .
$$

We remark that our previous arguments also give the following stronger result:

Proposition 4.1. For any irrational number $\alpha$ with $\mu(\alpha)<\infty$, if

$$
\sum_{j=1}^{t} \frac{1}{m_{j}} \rightarrow \infty \quad \text { as } x \rightarrow \infty
$$


then

$$
\max _{Y_{m}, X_{p}}\left|S\left(x \mid \alpha ; Y_{m}, X_{p}\right)\right|=o_{x}(1) \sum_{j=1}^{t} \pi\left(\frac{x}{m_{j}}\right) .
$$

Acknowledgements. The author would like to thank Prof. Xiumin Ren, Prof. Jianya Liu and Prof. Wenguang Zhai for their instructions and encouragement. The author is grateful to the referee for his comments and suggestions.

\section{References}

[1] H. Davenport, Multiplicative Number Theory, 2nd ed., Springer, 1980.

[2] K. H. Indlekofer and I. Kátai, Some remarks on trigonometric sums, Acta Math. Hungar. 118 (2008), 313-318.

[3] I. Kátai, A remark on trigonometric sums, ibid. 112 (2006), 221-225.

[4] K. F. Roth, Rational approximations to algebraic numbers, Mathematika 2 (1955), $1-20$.

School of Mathematics and System Sciences Shandong University

Jinan, Shandong, 250100, P.R. China
School of Mathematical Sciences Shandong Normal University Jinan, Shandong, 250014, P.R. China

E-mail: laohuixue@sina.com

Received on 19.10.2007

and in revised form on 25.1.2008 